Mots. Les langages du politique

Dire les minorités linguistiques en sciences

sociales : les notions de « vitalité » et d'« allophone » dans les contextes canadien et français

Wording linguistic minorities in the social sciences: the concepts of "vitality" and "allophone" within Canadian and French contexts

Hacer referencia a las minorías lingüísticas en las ciencias sociales: los conceptos de «vitalidad» $y$ «alófono» en los contextos de Canadá y Francia

Elatiana Razafi et Christophe Traisnel

\title{
OpenEdition
}

Journals

Édition électronique

URL : http://journals.openedition.org/mots/22888

DOI : $10.4000 /$ mots. 22888

ISSN : 1960-6001

Éditeur

ENS Éditions

Édition imprimée

Date de publication : 1 novembre 2017

Pagination : 111-126

ISSN : 0243-6450

Référence électronique

Elatiana Razafi et Christophe Traisnel, «Dire les minorités linguistiques en sciences sociales : les notions de "vitalité » et d'« allophone » dans les contextes canadien et français », Mots. Les langages du politique [En ligne], 115 | 2017, mis en ligne le 21 novembre 2019, consulté le 05 janvier 2021. URL http://journals.openedition.org/mots/22888 ; DOI : https://doi.org/10.4000/mots.22888 


\section{Dire les minorités linguistiques en sciences sociales : les notions de " vitalité " et d' " allophone " dans les contextes canadien et français}

\section{Introduction}

Le chercheur ${ }^{1}$ en sciences sociales n'est pas, loin s'en faut, qu'un observateur impartial du monde qui l'entoure (Berger et Luckmann, 1966). Comme tout acteur social, il fait face à la circularité des notions qu'il utilise, emprunte, fabrique. A ce titre, les «pères fondateurs» de la sociologie offrent des balises claires. Durkheim (1988 [1894]) demande en effet de découper (au sens d' " isoler ») l'objet visé et propose un processus scientifique rigoureux, objectif, à travers lequel la phase initiale est, précisément, de s'extraire systématiquement de toute prénotion ou préjugé pour mieux parvenir à l'étude du «fait social». Prendre un fait social «comme une chose» implique un travail de définition méthodique à travers lequel le contexte doit avoir le moins d'emprise possible. Quant à Max Weber (2003 [1919]), s’il reconnait lui-même (pour avoir tout à la fois été lui-même engagé et savant) que la distanciation s'avère difficile, le savant doit bien prendre la mesure des buts très distincts et des conditions très différentes de l'exercice du métier de savant et de celui du politique, l'un et l'autre ne reposant pas sur la même "éthique », ni ne partageant pas la même vocation. Éthiques de conviction et de responsabilité ne sont pas mobilisées de la même manière par l'un et par l'autre. Dans les deux cas, il s'agit d'opérer une (saine et rigoureuse) distanciation nécessaire à l'objet du métier de savant, à travers sa contribution au «désenchantement du monde » (Weber, 2003 [1919]). Le rôle ainsi assigné au chercheur est de se « déprendre» de ces contraintes pour établir des faits dits «scientifiques». Dès lors, le chercheur qui souhaite saisir ce qui fait sens pour lui, réengager explicitement la

1. La valeur générique du masculin grammatical est adoptée par défaut.

Université de Nouvelle-Calédonie

elatiana.razafi@univ-nc.nc

Université de Moncton, École des hautes études publiques

christophe.traisnel@umoncton.ca 
part interprétative de son travail ou encore revendiquer sa propre subjectivité ne risque-t-il pas de réenchanter le monde? La construction de «minorités sociales» illustre de manière intéressante ce type de tension.

Dans la mesure où un « groupe social n'est jamais minoritaire en soi» (Voutat et Knuesel, 1997, p.137) et en souscrivant à l'idée selon laquelle «c'est l'individu et non le concept qui est la source des conceptions valides sur le social» (Herman, 1983, p.46), le chercheur doit effectivement prendre en compte des facteurs plus largement contextuels et auxquels il participe nécessairement s’il veut comprendre les processus de minorisation. Récursivement, les paramètres de recherche ${ }^{2}$ qu'il définit agissent sur la manière dont lesdites minorités sont mises en perspective (Giddens, 1984) mais aussi représentées dans l'espace public. Les choix discursifs du chercheur interviennent dans la construction des minorités sociales et de leurs (auto)représentations parce que son discours est mobilisable par d'autres acteurs. Dans un tel mouvement circulaire, des notions s'imposent au chercheur tout autant qu'il impose les siennes aux autres. Toute mise en discours reflète donc des choix plus ou moins conscientisés, interrogés, assumés. Or, toute forme de dénomination induit une vision de la relation à l'autre et celles adoptées dans des dispositifs institutionnels jouissent d'une visibilité importante accréditée d'emblée d'un capital de légitimité. De ce fait, la reprise ou non des modes de désignation des minorités sociales par le chercheur mérite un questionnement.

C'est dans cette optique que nous nous intéresserons ici aux notions de «vitalité » et d' "allophone». Ces deux notions, dans deux contextes (Canada3 et France), sont en effet exemplaires de cette difficile déprise du chercheur face aux modes de désignation dominants. La première, "vitalité », rapportée aux communautés francophones minoritaires est rattachée au dialogue étroit entre «majorités» et «minorités » linguistiques au Canada. La notion de «vitalité» sera abordée tout à la fois à travers son usage au sein de la législation et des politiques de reconnaissance destinées aux «Communautés de langues officielles en situation minoritaire » (CLOSM), mais également au sein de la littérature scientifique consacrée à ces CLOSM au Canada. Les discours du monde scolaire nous intéressent en ce que l'école représente le secteur le plus visiblement investi par les politiques linguistiques. Plus largement, l'école donne aussi à voir comment une société conçoit l'altérité sociale. L'école constitue aussi le terrain d'observation ethnographique à partir duquel la seconde notion, «allophone», sera problématisée. La notion d'«allophone » fait écho à l'appellation actuellement en vigueur en France : «Enfants allophones nouvellement arrivés» (EANA). Face à cela, quelles alternatives discursives s'offrent

2. Relatifs au terrain, aux méthodes d'observation et d'enquête, aux modalités d'intervention, aux positionnements épistémologiques, etc.

3. Cette désignation géopolitique générale ne doit pas occulter que l'utilisation du terme «allophone » diffère entre le Québec et les autres communautés francophones au Canada. 
au chercheur soucieux de valoriser la pluralité linguistique et culturelle des minorités sociales en question?

\section{La notion de « vitalité » au Canada}

Il n'est pas utile ici de revenir sur l'histoire de la question nationale au Canada, mais simplement de rappeler que le contexte linguistique a longtemps été caractérisé par «deux solitudes» (MacLennan, 1945) caractérisant deux communautés linguistiques : le Canada français d'une part, francophone et catholique, et le Canada anglais, anglophone et protestant. Une double solitude dans laquelle, selon une interprétation donnée, l'ancienne majorité de langue française a progressivement subi, suite à la conquête par la Grande-Bretagne de l'Amérique française, un processus de minorisation, ponctué de tentatives très claires d'assimilation (allant parfois jusqu'à l'interdiction de la scolarisation en français), posant non moins clairement la question de la «survivance » du groupe francophone, jusqu'à l'affirmation plus ou moins sonore de celui-ci à travers la contestation politique portée par l'indépendantisme québécois des années 1960. Il s'agissait pour les élites canadiennes-françaises de mettre en cause un rapport de domination se traduisant notamment par l'entrave à l'enseignement en français, des inégalités de revenus entre groupes linguistiques, et plus généralement une difficulté à pérenniser l'usage d'une langue et sa transmission dans un contexte où le français ne disposait pas d'un statut officiel, à parité avec l'anglais. Le danger couru d'un éclatement du Canada par une forme de «convergence des luttes» de la question nationale québécoise et de la question linguistique dans les années 1960 a conduit le gouvernement fédéral à repenser les fondements identitaires du Canada à travers une approche davantage duale notamment de la langue et des politiques linguistiques. C'est le sens de la mise sur pied en 1963 de la Commission Laurendeau-Dunton sur le «bilinguisme et le biculturalisme». Ce contexte historique, trop rapidement esquissé ici, doit être pris en compte pour comprendre par la suite le succès de la notion de «vitalité». Une notion qui s'inscrit, finalement, dans cette préoccupation récurrente de la survivance qui a taraudé les élites canadiennes-françaises, puis francophones et québécoises au point de s’imposer comme notion charnière dans la littérature consacrée à la francophonie en situation minoritaire au Canada.

Les travaux de la Commission ont en effet permis de poser dès 1969 les jalons d'une législation sur le bilinguisme officiel avec l'adoption de la Loi sur les langues officielles. Plusieurs dispositifs ont accompagné cette législation : création du Commissariat aux langues officielles, constitution d'un fond de contestation judiciaire4, initiatives publiques dans plusieurs secteurs réputés cruciaux quant à l'épanouissement des communautés francophones

4. Permettant, en cas de non respect de la loi, de financer les actions devant les tribunaux. 
(éducation et culture, mais aussi santé, médias, développement économique, justice et immigration). Par la suite, ce cadre légal s'est trouvé renforcé d'abord par l'inscription des droits linguistiques dans la Charte canadienne des droits et libertés entrée en vigueur en 1982, ensuite par l'adoption d'une nouvelle mouture de la Loisur les langues officielles en 1988 fixant un certain nombre d'obligations du gouvernement à l'égard des «Communautés de langues officielles en situation minoritaire » au Canada, obligations rendues par la suite exécutoire, la Partie VII de cette loi obligeant le gouvernement à mettre en place des mesures positives à même d'assurer le développement de ces CLOSM. La législation a notamment prévu dans ses dispositions la mise en œuvre de «mesures positives » à l'égard des CLOSM, et le caractère obligatoire de ces mesures positives, notamment en matière d'éducation en français. Sous le coup de cette contrainte, en termes de moyens plus que de résultats que le gouvernement fédéral s'est lui-même imposé, plusieurs dispositifs ont été élaborés et des politiques à destination des CLOSM lancées, notamment à travers plusieurs plans d'action et feuilles de route, avec l'objectif d'améliorer cette vitalité. Dès lors, c'est une véritable politique de reconnaissance linguistique qui s'est mise en place au Canada, et dont l'un des objectifs est, selon les termes mêmes de la loi de 1988, de "favoriser l'épanouissement des minorités francophones et anglophones du Canada et à appuyer leur développement »5.

Dans ce cadre, la recherche constitue un élément stratégique, tout à la fois dans l'élaboration de politiques publiques adaptées à l'objectif «vitalitaire » du gouvernement, mais également dans l'évaluation des résultats de ces politiques comme du caractère effectivement « positif» des mesures mises en place. Dès lors, le terme revêt une importance cardinale, car il constitue bel et bien le cadre même que s'est fixé l'État dans sa mission à l'égard des CLOSM en général et des «Communautés francophones en situation minoritaire » (CFSM) en particulier (deux autres notions issues elles aussi de la législation sur les langues officielles). Cette notion et la réalité sociale et communautaire à laquelle elle peut renvoyer constituent d'une certaine manière le passage obligé pour saisir la portée et les limites des obligations du gouvernement fédéral à l'égard des minorités de langues officielles. Elle a donc, pour les différents acteurs politiques ou communautaires, une dimension opératoire de première importance, qu'il s'agit de questionner, d'explorer, de comprendre.

L'usage de la notion de «vitalité » dans la littérature sur les minorités linguistiques au Canada ne date bien sûr pas de l'introduction de cette notion dans la version de 1988 de la loi sur les langues officielles, mais une telle intervention a pour le moins transformé les approches, en dynamisant les travaux portant sur la vitalité, et surtout en mettant sous les feux de la rampe la question, désormais

5. «Enhancing the vitality of the English and French linguistic minority communities in Canada and supporting and assisting their development » (c'est nous qui soulignons). 
cruciale, de l'évaluation de l'efficacité des politiques publiques linguistiques, comme des initiatives communautaires suscitées par les possibilités politiques offertes aux acteurs communautaires par la puissance publique.

Cette notion de "vitalité », pour le moins anthropomorphique et organiciste (ce qui est toujours très gênant en sciences sociales) avait été explorée ailleurs par la littérature sur les minorités dès les années 1960, dans la foulée des réflexions de Saussure sur la nécessaire présence, pour toute langue, et notamment pour celles en contact avec une autre, d'une «masse parlante» (Carli, 2007). C'est le cas du dialectologue Corrado Grassi qui, en 1969, cherche à définir la vitalité d'une langue en fonction de sa capacité d'innovation progressive au contact d'autres langues et qui évoque cette notion de «vitalité linguistique» (Carli, 2007). Au Canada également, on retrouve des auteurs qui utilisent la notion, comme H. Giles, R. Bourris et D. Taylor. Dans leurs travaux, ils définissent dès 1977 la vitalité d'un groupe ethnolinguistique comme «ce qui fait que ce groupe est susceptible de se comporter comme une entité collective distincte et active en situations intergroupes ». Dans cette optique, c'est tout un courant d'analyse qui s'est constitué autour de la question des facteurs, notamment sociodémographiques, susceptibles d'assurer à un groupe linguistique (vitalité ethnolinguistique) ou à la pratique d'une langue (vitalité linguistique) son maintien dans un espace social donné, en dépit d'une situation diglossique. Dans ce cadre, l'importance quantitative du groupe, sa répartition sur le territoire, sa densité, et sa capacité de transmission intergénérationnelle de la langue constituent les indices centraux d'une telle «vitalité ».

Cette approche vitalitaire n'est pas la seule à avoir abordé la question de la pérennisation d'une minorité linguistique en Amérique du Nord et des conditions de cette pérennisation. On peut notamment repérer deux autres approches qui s'intéressent moins aux dimensions sociodémographiques en tant que telles qu'aux dimensions identitaires ou politico-institutionnelles caractérisant les minorités en question. L'approche identitaire cherche à dépasser le simple enjeu linguistique en le considérant comme un des facteurs de distinction d'un groupe, constitutif d'une «petite société ». Cette approche cherche aussi à se questionner plus généralement sur les fondements sociohistoriques du groupe en question (Giles et al., 1977; Thériault, 1995; Bock, 2004 ; Martel, 1997 ; Heller, 1998 ; Langlois et Letourneau, 2004), en abordant le rôle des autres facteurs culturels entrant en jeu dans la définition d'une «identité collective». Une autre approche, juridico-politique, s'est développée à mesure que se précisait un véritable statut linguistique à travers l'intervention de l'État. En amont de cette intervention, il s'agissait d'imaginer des formes plus ou moins radicales d'autonomie politique à même d'institutionnaliser la minorité francophone à travers notamment une territorialisation de sa reconnaissance politique. C'est, d'une certaine manière, l'approche dualiste défendue par la Commission Laurendeau-Dunton. En aval de l'intervention 
fédérale, les analyses porteront plus généralement sur une évaluation du statut conféré aux CFSM par les politiques de reconnaissance linguistique, et à la portée ainsi qu'aux limites de ces politiques publiques (Calvet, 1986 ; Lapierre, 1988), des droits linguistiques (Werlin, 2005; Kymlicka et Patten, 2003) et des pratiques de gouvernance communautaire Johnson, 2003; Cardinal et Juillet, 2005; Forgues, 2003). Le statut de la langue est questionné (Johnson et Doucet, 2006 ; Taylor, 1994 ; Kymlicka, 1995; Tully, 1997).

Le champ de la recherche sur les minorités linguistiques au Canada a donc notablement évolué en même temps que les politiques linguistiques se sont mises en place (Bourgeois et al., 2006). Avec comme objectif d'évaluer «la vitalité des communautés de langues officielles en situation minoritaire», M. L. Johnson et P. Doucet dressent en 2006 une revue de la littérature sur la notion. À part quelques recherches avant-gardistes (notamment celles de Réal Allard et Rodrigue Landry en 1987), l'essentiel des titres cités par ce rapport sont bien postérieurs à la fin des années 1980 .

Cela étant, la littérature a vu plutôt s'imposer le terme «vitalité » tel qu'il figurait dans la traduction anglaise («vitality»), plutôt que celui, sans doute moins comptable et plus aléatoire, d' "épanouissement linguistique des minorités» cité plus haut. Auparavant, le terme «vitality», à la différence de celui d' «épanouissement des minorités», avait déjà été investi par les études sur les minorités linguistiques, notamment au Canada (Johnson et Doucet, 2006). En somme, en choisissant «vitality», on s'oriente vers une dimension surtout évaluative et quantitative des locuteurs pour analyser, à travers ses dimensions sociodémographiques, la force politique et sociale d'un groupe, ou encore son statut, et évaluer également la légitimité de la revendication de ses acteurs. Au-delà d'une réflexion sur les déterminants sociaux pesant sur la minorité, ou le rôle des acteurs, les études s'orientent plutôt sur l'évaluation des groupes, leur nombre, leur proportion. D'ailleurs, les besoins en recherche s'orientent autour de l'enjeu, central, d'une définition des communautés (en fonction de la répartition territoriale, de la densité et du nombre de locuteurs) comme de ses besoins (en fonction des taux de transmission linguistique, un des facteurs généralement retenus pour évaluer la vitalité des communautés). Les acteurs communautaires comme les différents ministères concernés par l'obligation de prendre, dans le domaine des minorités de langues officielles, des mesures positives sont en effet en demande de recherche et de produits de recherche leur permettant de définir des «indicateurs de la vitalité » à même de leur permettre de remplir leur mission d'évaluation des politiques mises en œuvre6 ${ }^{6}$ Dans un tel contexte, de plus en plus d'études et de recherches s’inscrivent dans ce type de démarches,

6. La production de tels indicateurs se révèle en effet nécessaire dans le travail d'évaluation des différents programmes à destination des communautés de langues officielles en situation minoritaire. Dans ce travail, les chercheurs sont, là aussi, sollicités, de même que les consultants et experts en analyse des politiques publiques. 
à tel point qu'il est possible de parler, s'agissant de la recherche sur la francophonie canadienne, d'un véritable «paradigme vitalitaire », qu'on retrouve, par exemple, dans l'énoncé des axes de recherche prioritaires par les instituts, dans les «profils» des communautés produits par les organismes, ou dans les rapports commandés et diffusés par les institutions publiques7. Un tel paradigme se trouve renforcé par la capacité de recherche propre à certains ministères, agences publiques ou organismes communautaires. Le milieu s'est structuré, avec ses instituts spécialisés (ICRML, CIRCEM, CRCCF, etc.), ses revues (Minorités et sociétés, Francophonies d'Amérique, etc.), des sources de financement public de la recherche ou de production publique de recherche sur ces domaines (Statistique Canada; Commissariat aux langues officielles, Patrimoine Canadien etc.).

La législation, les dispositifs comme les politiques ont donc généré un «appel d'air» quant à la production de recherche et à l'analyse des transformations des CLOSM au Canada. Ce faisant, c'est également un appareil conceptuel et des référents propres à la gouverne canadienne qui se sont peu à peu imposés au monde de la recherche dans plusieurs domaines : commandites, avis d'experts devant les tribunaux dans le cadre de causes linguistiques visant à évaluer les besoins, l'état des services ou l'impact des mesures gouvernementales sur l'épanouissement des communautés; besoins des organismes communautaires eux-mêmes qui, dans chacun des domaines réputés "vitaux» pour les CLOSM (éducation, santé, culture, médias, développement économique, immigration), ont mis en œuvre avec le gouvernement des services adaptés dont il convient, là aussi, d'évaluer l'efficacité ou la pertinence.

Le milieu de la recherche en francophonie canadienne s'est donc transformé, la recherche étant très fréquemment mentionnée comme un des domaines stratégiques dans le renforcement de la vitalité des communautés. Si on s'intéresse à l'ensemble des recherches produites et fréquemment citées dans les revues de littératures produites sur les CLOSM (rapports publics, rapports de consultant, travaux universitaires, subventionnés ou non), on passe ainsi d'une recherche encore très marquée par les approches historiques et littéraires dans les années 1970, à une recherche plus identitaire et sociopolitique dans les années 1980 (à travers les travaux de Breton par exemple), pour opérer un tournant depuis la fin des années 1980 où c'est le «paradigme vitalitaire » qui désormais domine le champ de recherche sur les francophonies canadiennes.

Il apparait ainsi que l'accès à la connaissance des minorités francophones canadiennes demeure largement balisé par les grandes références

7. Mentionnons ici, à titre de simple illustration, l'un des trois axes de recherche de l'Institut canadien de recherche sur les minorités linguistiques (ICRML) intitulé «vitalité des communautés», ou les profils des communautés diffusés par la Fédération des communautés francophones et acadiennes du Canada (FCFA) et exposant les vitalités économique, démographique, linguistique ou plus largement communautaire, ou encore les rapports produits par le Commissariat aux langues officielles. 
institutionnelles, allant jusqu'à consacrer dans le milieu de la recherche, outre la référence à la «vitalité » linguistique ou communautaire elle-même, les références, utilisées ici à plusieurs reprises de «communautés de langues officielles en situation minoritaires» (CLOSM) ou de «communautés francophones en situation minoritaire» (CFSM). La notion d' «allophone» met également en exergue la nécessité de questionner plus systématiquement le fondement des modes de désignation dominants dans le domaine de la recherche sur les minorités.

\section{La notion d'«allophone » en France}

En France ${ }^{8}$, la dénomination des publics scolaires minoritaires s'est construite autour d'une pluralité de critères au gré des circulaires. Les focalisations visent tantôt le rapport au territoire - «nouveaux arrivants» (2002); «nouvellement arrivé en France» (2002 et 2012) -, tantôt le statut civil de l'élève - «élèves de nationalité étrangère » (2002) (voir aussi Galligani, 2012 ; France, 2013b ; Razafimandimbimanana, 2014). La mobilité et le caractère diasporique ont aussi donné lieu à des appellations telles que «enfant de migrants» (1986). Chaque appellation officielle est inscrite dans une temporalité sociale, historique, politique donnée et conséquemment, vouée à être remplacée. C'est ce qu'illustre la récente substitution, en octobre 2012, de l'appellation «Enfants nouvellement arrivés en France » (ENAF) par «Elèves allophones nouvellement arrivés» (EANA). À travers ce changement terminologique, la Direction générale de l'enseignement scolaire du ministère de l'Éducation nationale a mis en circulation un terme nouveau pour l'administration française 9 : «allophone ». La question de l'inclusion des parcours (pluri)linguistiques ainsi jugés différents par rapport à « une norme attendue et jugée seule légitime» (Castellotti, 2009), est souvent étudiée du point de vue des implications didactiques ${ }^{10}$. Qu'en est-il des conséquences sur les élèves qui s'en trouvent officiellement catégorisés en tant qu'allophones?

En statuant sur les publics concernés par l'organisation de la scolarité obligatoire, le ministère de l’Éducation nationale français dénomme et, conséquence de tout acte dénominatif, assigne un trait identitaire dominant aux différents groupes d'élèves. Ceux auxquels sont associés des profils et des parcours minoritaires sont prioritairement visés par les politiques d’intégration. Du fait de ce statut minoritaire, du traitement distinctif qui leur est des-

8. Pour un parcours critique des appellations rattachées au contexte scolaire québécois, voir notamment Razafimandimbimanana, 2008.

9. Précisons que le terme est opératoire dans le contexte de l'organisation scolaire au Québec depuis 1995 .

10. Le Centre de ressources et d'ingénierie documentaires du Centre international d'études pédagogiques propose par exemple une sélection de ressources traitant de La place de la langue maternelle des élèves allophones à l'école (février 2015); document disponible sur Internet : 〈http://www.ciep.fr/sites/default/files/atoms/files/focus-place-langue-maternelle-deseleves-allophones-a-l-ecole.pdf> (consulté le 14 avril 2017). 
tiné et des tensions qui se greffent à ces formes de différenciation sociale, il est aisé de voir en quoi l'acte dénominatif peut aussi relever d'une intervention aux effets sociaux et symboliques dévalorisants. Cette interdépendance entre les modes de désignation et les (auto)représentations des jeunes qu'ils catégorisent repose la question de l'inclusion scolaire, d'un point de vue sociosymbolique cette fois, pour savoir en quoi il serait pertinent pour le chercheur de prendre à son compte la notion officielle d' "allophone».

Il est évident que la question donne lieu à différentes réponses selon les situations interactionnelles, motivations sociales et facteurs de communication en jeu. Par exemple, un chercheur s'adressant à des décideurs politiques favorisera certainement l'intercompréhension en reprenant la terminologie en vigueur. Outre ce facteur strictement communicationnel, un choix consensuel peut aussi s'avérer stratégique en vue de mieux convaincre autrui. Dans tous les cas, la reprise de l'appellation institutionnalisée semble être un choix efficace et économique tant elle est répandue. Nombreux sont effectivement les acteurs du milieu éducatif et de la recherche qui parlent des EANA (et auparavant d'ENAF) et des «allophones». En dehors de contextes spécifiques contraignant la reprise des dénominations administratives, certains espaces d'interventions (échanges informels, colloques, publications) offrent une marge de manœuvre aux chercheurs tout en leur laissant la possibilité, si besoin est, d'expliciter les équivalents officiels. Envisager des alternatives suppose que l'appellation officielle n'est pas neutre et peut s'avérer contradictoire par rapport à une politique d'intégration.

Avant de considérer cette hypothèse, rappelons-en les fondements. Dans une vision optimiste, la notion d' "allophone " s'interprète comme un changement allant dans le sens d'une meilleure inclusion sociale par rapport aux expressions antérieures comme "élèves non-francophones». On peut également y voir une forme de reconnaissance des compétences plurilingues des élèves en question :

Là où l'appellation «non-francophone» envisageait avec un préfixe privatif l'élève nouvellement arrivé sous l'angle de la lacune, du manque à combler voire du handicap, l'appellation «allophone» met en avant le préfixe «allo» qui se réfère à la notion d'alter. Il s'agit donc de considérer l'élève allophone comme celui (ou celle) qui parle une autre langue ou qui parle d'autres langues. Il y a donc un renversement de considération car cette nomination présuppose des compétences dans des langues autres que le français. » (France, 2013b, p. 3 ; souligné dans l'original)

Indépendamment des appellations officielles qui se succèdent, l'objectif de l'administration française reste inchangé en ce qu'il s'agit toujours de tracer - de la manière la plus précautionneuse qui soit - les frontières entre la «norme » scolaire et le cas particulier. De ce point de vue, l'institutionnalisation du terme «allophone», dont les racines étymologiques renvoient à «allos» («autre» en ancien grec), ne peut que paraître cohérente. En même temps, il 
faut y voir une fonction euphémistique puisque le terme permet de désigner l' «autre», le «différent», l' «étranger», le «non francophone» voire le «non Français» sans pour autant le nommer comme tel. L'élève dont il est question est pourtant celui dont l'expérience scolaire est jugée temporairement insuffisante pour valoir une inscription au sein du système dit «ordinaire». Des dispositifs d'accueil, de scolarisation et d'accompagnement spécifiques lui sont donc destinés. En tant que locuteur de langue(s) autre(s) que le français, l'« allophone » est aussi l'élève qu'il faut franciser. Et c'est peut-être là un objectif institutionnel moins dicible/audible en France : celui de l'intégration sociale.

En même temps qu'elle réaffirme la volonté historique d'une identité nationale uniforme (et monolingue), cette idée de francisation fait effectivement réapparaître les traumatismes des défenseurs des langues régionales face à l'école républicaine, par ailleurs décriée comme organe de l'assimilation (mono) linguistique à partir de la Révolution française :

Les idéologues révolutionnaires, au premier rang desquels figuraient l'abbé Grégoire et [B.] Barère, dressent le constat d'un pays morcelé linguistiquement dont la cohésion implique l'universalisation de la langue française par l'école au détriment des «patois», perçus comme des obstacles à l'édification de la nation. (Cortier et Puren, 2008, p.64)

La notion d' "allophone » fait d'ailleurs écho à celle d' " alloglotte», utilisée pour désigner les populations visées à l'époque par la francisation en France et dans les colonies. Dans les deux cas, la notion d'altérité sert de principal critère d'identification. La question qui se pose en creux concerne la manière dont l'autre est socialement perçu.

Comme l'ont démontré nombre de travaux en sociologie, celui dont la place est définie par sa différence peut être positivement représenté comme étant un acteur social intégré (Simmel, 2009 [1908], p.54). C'est en ce sens que nous avons évoqué l'acceptation optimiste de la notion d' «allophone». Dans une vision plus pessimiste, l'autre est, au contraire, dépeint comme une figure menaçante à l'instar de l'analyse interactionniste suivante :

Lorsqu'il est perçu comme étant plus ou moins ignorant des rites de la société dans laquelle il se trouve et, par conséquent, susceptible de se conduire de façon imprévisible, l'étranger représente une menace pour la face de chacun et pour le bon déroulement de l'interaction en général. (Frame, 2013, p. 64)

Nous savons aussi qu'en accentuant l'altérité d'autrui, nous le réduisons à un statut d'étranger sans possibilité d'appartenance commune (Ricœur, 1990). Ce qui est d'autant plus plausible dans le cas des élèves dits «EANA» que l' «élève allophone est fantasmé comme irréductible et radical» (France, 2013 b, p. 5). En reprenant à son compte la notion d' "allophone» pour désigner des jeunes qui, par ailleurs, revendiquent leurs expériences plurilingues et pluriculturelles, le chercheur les expose donc à une assignation identitaire. 
Des récits d'expériences d'élèves qui se voient désignés montrent aussi comment le « regard des élèves francophones sur [le] statut d'élève allophone est méprisant ou ressenti comme tel» (Guedat-Bittighoffer, 2013, p.164). Ce qui rappelle les effets négatifs de l'ethnicisation. En psychologie sociale, ce processus consiste, pour un groupe majoritaire, «à voir les individus issus de l'immigration comme les membres d'une catégorie définie par une origine et une culture différente (de soi) et inférieure » (Lorcerie, 2007). Qu'elle soit institutionnelle ou pas, nous pensons que toute catégorisation sociale est sujette à l'ethnicisation et à l'exclusion sociale. Le piège des appellations officielles est sans doute d'objectiver les critères qu'elles retiennent. Nous y reviendrons.

Un autre problème se pose si nous considérons que la notion d' «allophone » désigne exclusivement «tout locuteur de langue maternelle autre que le français» et que, par voie de conséquence, "francophone» désigne «tout locuteur de langue maternelle française ». Ce qui renouvelle le statut mythique du locuteur «natif» comme pour naturaliser, objectiver, légitimer la différenciation sociale en jeu. Cette approche restrictive est opératoire au Canada où l'étanchéité des frontières entre les communautés linguistiques s'explique par une politique de reconnaissance formelle des groupes minoritaires et, en vue de son application, des besoins de recensement statistique. Théoriquement, un élève «allophone» ne peut donc devenir «francophone» au Canada. Nous avons pu observer cette même étanchéité dans les modes de désignations opératoires chez le personnel enseignant au sein d'établissements scolaires au Québec (Razafimandimbimanana, 2008). En France, la notion d' «allophone », devait «promouvoir la reconnaissance des compétences dont disposent ces élèves dans leur langue d'origine» (France, 2013a, p. 3) et contrer le regard dépréciatif que pouvait suggérer l'expression précédemment en vigueur «non francophone ». Les textes officiels reconduisent pourtant des conceptions nativistes et monolingues puisqu'ils définissent les publics scolaires «allophones» en fonction de leur «langue maternelle» ou «langue d'origine», encore pensées au singulier. Également, la conception des «EANA» reste ancrée dans une vision déficitaire en désignant les élèves arrivants « ne maîtrisant pas la langue de scolarisation » (France, 2012). L'appellation «allophone » est donc susceptible de voir l'identification des élèves se cristalliser autour d'appartenances sociales et linguistiques uniques, exclusives et lacunaires.

Enfin, le terme "allophone» souffre de ses propres implicites dès lors où il est censé désigner l'ensemble hétérogène des communautés linguistiques autres que francophones (au sens strict). II recouvre également une pluralité de profils, parcours et biographies langagières. Ce sont ces différentes expériences et compétences plurielles qui, derrière le seul vocable d' «allophone », sont potentiellement ignorées d'autant que les dimensions plurilingues et pluriculturelles des élèves figurent rarement dans les discours et représentations des enseignants (Galligani, 2012). Sachant cela, le chercheur peut donc 
agir en faveur d'une meilleure reconnaissance de ces expériences et compétences là en refusant de les «euphémiser». Dans le cadre de travaux menés au sein de dispositifs d'accueil en France (mais également au Québec), nous faisons ainsi le choix de parler de l'élève dans son ensemble en préférant le terme «plurilingue » à celui, axé sur une compétence absente, d' "allophone». Dans l'argumentation autour de ce choix (voir Razafimandimbimanana, 2008 et 2014), retenons qu'il ne peut prétendre échapper ni à la construction d'autres inégalités sociales, ni à l'ethnicisation (voir supra et Lorcerie, 2007). Les observations que nous avons pu effectuer ont d'ailleurs permis de constater que souvent, la conscience de la différence sociale prévalait sur celle d'une appartenance commune. L'emploi du terme «plurilingue» est néanmoins pensé pour agir sur ces (auto) représentations limitatives en faisant de la maîtrise de plusieurs langues, trait potentiellement valorisant, la base d'une différenciation positive. À l'inverse, la notion d' «allophone» prive les élèves plurilingues de l'explicitation de leurs expériences plurilingues et pluriculturelles, celles-là même qui pourraient justement être transposées en compétences scolaires et qui devraient l'être. Cela devient une nécessité quand on connaît :

1/ le parti pris monolingue avec lequel s'effectue traditionnellement l'étude du bilinguisme (Grosjean, 1984, p.17) et, par extension, la pluralité linguistique ;

2/ les représentations de «l'élève allophone [qui] est toujours considéré comme un apprenant monolingue» (Guedat-Bittighoffer, 2013, p.162);

3/ le peu de place accordée à la pluralité linguistique en contexte scolaire en France (Razafimandimbimanana, 2014).

En utilisant le terme "allophone» pour désigner un élève plurilingue, on risque ainsi d'oublier (ou de faire oublier) qu'il s'agit d'un élève possédant de réelles compétences communicatives dans plusieurs langues y compris en français, langue en cours d'appropriation. Vue exclusivement à travers le statut d' "allophone », la situation linguistique de l'élève est effectivement appréhendée de manière lacunaire avec, encore une fois, une focalisation sur la langue du pays d'accueil. Enfin, le sort de l'«allophone» dépend de la plasticité prêtée aux modes de catégorisation. Par exemple, au Québec, les «allophones» ne deviendront jamais, au sens technique du terme (Béland, 1999, p. 1), «francophones» (Razafimandimbimanana, 2008, p. 252).

Nous n'avons pu traiter, jusque-là, du phénomène annexe de l'institutionnalisation de catégories sociales. Il nous semble important de rappeler qu'en tant qu'instrument du groupe majoritaire, l'institutionnalisation participe au maintien de l'ordre social pensé par et pour le groupe majoritaire. Il traduit donc le projet social du majoritaire tout en objectivant et légitimant ses conditions de différenciation/domination sociale. Et nous savons que la légitimation est une dimension essentielle à tout processus de construction sociale en en justifiant l'existence (Berger et Luckmann, 1966, p. 79). 
Sous cet angle, l'appellation "EANA», officiellement nouvelle depuis octobre 2012, ne fait que réinvestir des marqueurs identitaires déjà communément admis : statut socioprofessionnel (élève), profil linguistique (allophone) et rapport au territoire (nouvellement arrivés). Implicitement, elle réitère que l'appartenance sociale est soumise à des conditions de performances socioprofessionnelles, linguistiques en français et à une présence durable sur le territoire. Il serait difficile de contester la pertinence de ces critères tant ils ont été intériorisés comme garants d'une identité nationale crédible. Cyniquement, la question des rapports aux appellations institutionnelles reviendrait en quelque sorte à choisir quelles croyances collectives nous partageons et auxquelles nous renonçons.

\section{Perspectives : parad oxes autour du rôle du chercheur}

Les notions de "vitalité » et d' "allophone» se sont peu à peu imposées dans les champs d'étude des langues au Canada et en France. Les organes des États respectifs s'impliquent, ils légifèrent, créent des dispositifs autour des langues « officielles » ou des publics concernés par la francisation. Les États interviennent également au sein des processus de production scientifique à travers, entre autres, des financements, évaluations de politiques et de programmes, commandes publiques. Il en résulte non seulement une intervention dans la définition des actualités et des priorités de la recherche mais également dans la manière de conceptualiser les sujets de recherche. Ainsi, au Canada, l'approche «vitalitaire » est devenue paradigmatique autour des enjeux des minorités/majorités linguistiques, de langues officielles, de bilinguisme, de communautés de langues officielles en situation minoritaire et de communautés francophones en situation minoritaire. Les organismes statistiques imposent aussi leurs cadres et critères de recensement dans l'identification des groupes sociaux. Des cloisons identitaires sont ainsi parfois construites entre les catégories «francophones", " anglophones» «allophones » ou même "bilingues». En France, la reprise de l'acronyme EANA et de la notion «allophone » réifie et banalise des catégorisations initialement conçues pour des contextes administratifs. Nous préférons en effet parler de jeunes "plurilingues » afin de mieux valoriser les expériences et compétences plurielles des acteurs en question.

Souvent malgré lui promoteur et contestataire des modes dominants de désignation des minorités, le chercheur semble jouer un rôle paradoxal : ses rattachements institutionnels le contraignent, il est lui-même habité par ses propres motivations (sociales, politiques, épistémologiques, éthiques, etc.) tandis qu'il se voit historiquement et communément associé à l'idéal du scientifique objectif (Daston et Galison, 2012). En empruntant quelque peu à l'anthropologie, nous héritons aujourd'hui de trois conceptions différentes du 
rôle du chercheur qui s'entrechoquent (Sperber, 1981) : celle où sa fonction est d'observer, de décrire et d'expliquer les faits «naturels» de la société (Radcliffe-Brown, 1952); celle selon laquelle la description produite intègre ses interprétations (Evans-Pritchard, 1962) et celle qui soutient que la description et l'explication relèvent précisément de l'interprétation (Geertz, 1973).

Ces débats inhérents à l'histoire des sciences permettent d'illustrer le poids des paradoxes autour du rôle de «chercheur». Au-delà des cadres de référence dont il se saisit, le chercheur est contraint par des contextes enchâssés en même temps qu'il est responsable des effets que produisent ses choix sur ces mêmes contextes. Cela revient à dire que le chercheur ne peut prétendre évoquer une forme de neutralité bienveillante lorsqu'il désigne l'autre dans l'exercice de son métier.

\section{Références}

ALLARD Réalet, LANDRY Rodrigue, 1987, «Contact des langues, vitalité linguistique subjective et comportement ethnolangagier ", Langage et société, n 41, p. 83-84.

Beland Paul, 1999, Le Français, langue d'usage public au Québec 1977, Rapport de recherche, Conseil de la langue française, http://www.cslf.gouv.qc.ca/publications/ pubb148/formatpdf/b148.pdf (consulté le 13 février 2017).

Berger Peter L. et Luckmann Thomas, 1966, The Social Construction of Reality. A Treatise in the Sociology of Knowledge, New York, Doubleday.

Bock Michel, 2004, Quand la nation débordait les frontières. Les minorités françaises dans la pensée de Lionel Groulx, Montréal, Éditions Hurtubise HMH.

Bourgeois Daniel, Denis Wilfied, Dennies Donald et Johnson Marc L., 2006, La Contribution des gouvernements provinciaux et territoriaux à l'épanouissement des communautés francophones en milieu minoritaire : un premier bilan et quelques prospectives, Rapport de recherche, Moncton, ICRML.

BRETON Raymond, 1983, "La communauté ethnique, communauté politique », Sociologie et sociétés, vol. 15, n² 2, p. 23-38.

CALVET Louis-Jean, 1986, "Typologie des politiques linguistiques», États de langue : Peut-on penser une politique linguistique?, M.-P. Gruenais éd., Paris, Fayard, p. 15-47.

CARDINAL Linda et JUILLET Luc, 2005, «Les minorités francophones hors-Québec et la gouvernance des langues officielles au Canada », La Gouvernance linguistique : le Canada en perspective, J.-P. Wallot éd., Ottawa, Presses de l'Université d'Ottawa, p.157-176.

CARLI Augusto, 2007, "Le concept de "vitalité linguistique" à l'exemple des “langues minoritaires", des "langues moins utilisées" et des "langues majoritaires" ", Communication à la Conférence de l'European Federation of National Institutions for Language (EFNIL), Language Policies in Europe, Riga; disponible sur Internet \http://www. efnil.org/documents/conference-publications/riga-2007/Riga-11-Carli-Mother.pdf> (consulté le 13 avril 2017).

CAStellott Véronique, 2009, "Construire l'intégration en (dés)intégrant les catégories? », Le Français aujourd'hui, n0164, p. 109-114.

CORTIER Claude et PUREn Laurent, 2008, «Français et langues régionales et/ou minoritaires : une mise en convergence difficulteuse », Repères. Recherches en didactique du français langue maternelle, $\mathrm{n}^{\circ} 38$, p. 63-80. 
DAston Lorraine et Galison Peter, 2012, Objectivité, Dijon, Les Presses du réel.

DURKHEIM Emile, 1988 [1894], Les Règles de la méthode sociologique, Paris, Flammarion.

EVANS-PRITCHARD Edward, 1962, Essays in Social Anthropology, Londres, Faber.

FORGUES Éric, 2004, Capital social, gouvernance et rationalisation des pratiques communautaires; outils théoriques et méthodologiques, Rapport de recherche, Moncton, Institut canadien de recherche sur les minorités linguistiques; disponible sur Internet <http://www.icrml.ca/fr/recherches-et-publications/publications-de-l-icrml/ download/96/8481/47> (consulté le 13 avril 2017).

Frame Alexander, 2013, Communication et interculturalité: Cultures et interactions interpersonnelles, Paris, Hermès Sciences/Lavoisier.

France, ministère de l'Éducation nationale, Direction générale de l'enseignement scolaire, 2012, Organisation de la scolarité des élèves allophones nouvellement arrivés, Circulaire no 2012-141 du 2-10-2012, NOR: REDE1236612.

France, académie d'Orléans-Tours, Centre académique pour la scolarisation des nouveaux arrivants et des enfants du voyage (CASNAV), 2013a, La Scolarisation d'un élève allophone nouvellement arrivé en classe ordinaire en élémentaire, Guide pédagogique mis à jour et complété, mis en ligne le 18 mars 2013 par Jacques Chavanes; disponible sur Internet <http://crdp2.ac-rennes.fr/blogs/fls/files/2012/03/Guide-descolarisation-des-EANA-en-classe-ordinaire.pdf〉 (consulté le 13 avril 2017).

France, ministère de l'Éducation nationale, Direction générale de l'enseignement scolaire, 2013b, L'Inclusion scolaire des EANA : questions d'éthique, de politique institutionnelle et de pratiques didactiques, Conférence de Cécile Goï et Delphine Bruggeman, juillet 2013; disponible sur Internet «http://cache.media.eduscol.education.fr/ file/FLS/01/6/conference_Goi_Bruggeman_263016.pdf> (consulté le 13 avril 2017).

GALLIGANI Stéphanie, 2012, «Regards croisés sur les enfants venus d'ailleurs et scolarisés en France ", Les Cahiers du GEPE, $\mathrm{n}^{\circ} 4$ (Les langues des enfants «issus de l'immigration » dans le champ éducatiffrançais, D. Huck éd.); disponible sur Internet 〈http:/ / www.cahiersdugepe.fr/index.php?id=2314〉 (consulté le 13 avril 2017).

GeERTZ Clifford, 1973, The Interpretation of Cultures, New York, Basic Books.

GIDDEns Antony, 1984, The Constitution of Society, Cambridge, Polity Press.

GILES Howard, BouHRIS Richard Y. et TAYLOR Donald M., 1977, «Towards a Theory of Language in Ethnic Group Relations », Language, Ethnicity and Intergroup Relations, G. Howard éd., Londres, Academic Press, p. 307-348.

GrosJeAn François, 1993, «Le bilinguisme et le biculturalisme : essai de définition », Tranel, $\mathrm{n}^{\circ}$ 19, p. 13-41; disponible sur Internet <https://www.unine.ch/files/live/sites/ islc/files/Tranel/19/Grosjean_13-41.pdf> (consulté le 13 avril 2017) [numéro intégral disponible à cette adresse : 〈https://www.unine.ch/islc/home/tranel/tousles-numeros/tranel-19.html']

Guedat-Bittighofer Delphine, 2013, «Récits d'expérience d'élèves allophones en classe ordinaire au collège en France : entre intériorisation douloureuse de la norme scolaire et rejet de son identité », Vers le Plurilinguisme? Vingt ans après, V. Bigot, A. Breteigner et M. Vasseur éd., Paris, Éd. des Archives contemporaines, p.150-165. Heller Monica, 1998, Linguistic Minorities and Modernity. A Sociolinguistic Ehnography, Londres-New York, Longman.

Herman Jacques, 1983, Les Langages de la sociologie, Paris, PUF.

JOHNSON Marc, 2003, "Agir sur la langue et être par la langue : les enjeux de la politique linguistique canadienne», Colloque international sur l'écologie des langues organisé par le Centre de recherche en linguistique appliquée de l'Université de Moncton, 
à Memramcook, Nouveau-Brunswick, du 21 au 23 août 2002, A. Boudreau, L. Dubois, J. Maurais et G. O’Connell éd., Paris, L'Harmattan, p.185-201.

Johnson Marc L. et Doucet Paule, 2006, Une vue plus claire : évaluer la vitalité des communautés de langue officielle en situation minoritaire, Ottawa, Commissariat aux langues officielles.

KYMLICKA Will, 1995, Multicultural Citizenship : A Liberal Theory of Minority Rights, Oxford, Clarendon Press.

Kymlicka Will et Patten Allan éd., 2003, Language Rights and Political Theory, New York, Oxford University Press.

LANG LOIS Simon et LÉTOURNEAU Josselin éd., 2004, Aspects de la nouvelle francophonie canadienne, Québec, Presses de l'Université Laval.

LAPIERRE Jean-William, 1988, Le Pouvoir politique et les langues : Babel et Léviathan, Paris, PUF.

LORCERIE Françoise, 2007, «Intégration, ethnicisation », Écarts d'identité, n ${ }^{111}$, p. 49-51. MaClennan Hugh, 1945, Two Solitudes, Toronto, Collins.

MARTEL Marcel, 1997, Le Deuil d'un pays imaginé. Rêves, luttes et déroute du Canada français. Les relations entre le Québec et la francophonie canadienne, 1867-1975, Ottawa, University of Ottawa Press.

RAdCLIFfe-Brown Alfred R., 1952, Structure and Function in Primitive Society, Glencoe, The Free Press.

RAZAFIMANDIMbIMANANA Elatiana, 2014, "Beyond "Culture”: Nationalistic and Monolinguistic Ideologies in Quebec », Cultural Essentialism in Intercultural Relations, F. Dervin et R. Machart éd., New York, Palgrave Macmillan, p.121-152.

-, 2008, Langues, représentations et intersubjectivités plurielles: une recherche ethnosociolinguistique située avec des enfants migrants plurilingues en classe d'accueil à Montréal, Thèse de doctorat, Sciences du langage, Sociolinguistique, Université de Haute-Bretagne-Rennes 2 ; disponible sur Internet «https://halshs.archives-ouvertes. $\mathrm{fr} /$ tel-00306026/document> (consulté le 13 avril 2017).

Ricceur Paul, 1990, Soi-même comme un autre, Paris, Éd. du Seuil.

Simmel Georg, 2009 [1908], «Digression sur l'étranger », L'École de Chicago. Naissance de l'écologie urbaine, Y. Grafmeyer et I. Joseph éd., Paris, Aubier-Flammarion, p.53-77 [Reproduction en fac-similé de l'édition de 1979].

SPERBER Dan, 1981, "L'interprétation en anthropologie ", L'Homme, vol. 21, nº1, p. 69-92.

TAYLOR Charles, 1994, Multiculturalism. Examining the Politics of Recognition, Princeton, Princeton University Press.

ThÉRIAULt Joseph-Yvon, 1995, L'Identité à l'épreuve de la modernité. Écrits politiques sur l'Acadie et les francophonies canadiennes minoritaires, Moncton, Éd. d'Acadie.

Tully James, 1997, Strange Multiplicity, Constitutionalism in an Age of Diversity, Cambridge, Cambridge University Press.

VoutAT Bernard et KNUESEL René, 1997, «La question des minorités. Une perspective de sociologie politique », Politix, n ${ }^{3}$ 8, p.136-149.

Weber Max, 2003 [1919], Le Savant et le politique : une nouvelle traduction. La profession et la vocation de savant. La profession et la vocation de politique, Paris, La Découverte.

WoEHRLING José, 2005, «Conflits et complémentarités entre les politiques linguistiques en vigueur au Québec, au niveau fédéral et dans le reste du Canada », Appartenances, institutions et citoyenneté, P. Noreau et J. Woehrling éd., Montréal, Wilson et Lafleur, p. 295-319. 